\title{
Preprints and Medical Journals: Some Things You Should Know as an Author
}

One of the hallmarks of the COVID-19 era is the explosion of preprint publications, forcing journals to establish or change their preprint policies. This is especially true for medical journals, and the Balkan Medical Journal is one of the journals affected by this trend.

Due to the recent submission of preprints, we have had to add a preprint policy to our editorial policy and author guidelines for the Balkan Medical Journal. In this issue, for the first time, we are publishing a study that was previously posted to a preprint platform.

We received an article a few months ago, but the author had not informed us that the article had been sent to a preprint platform before it was sent to our journal. Before sending the manuscript to the reviewers, we checked its similarity score, and noticed that the similarity rate was extremely high. We found that this was due to the preprint version of the same manuscript. This experience led us to decide that we need a preprint policy and that the journal should guide the authors in this regard. This is why we created our preprint policy and published it on the web, in consultation with our editorial board. The full policy is now available online at https:// balkanmedicaljournal.org/en/editorial-policy-1018. Now, through this editorial, we wish to inform the authors and readers about preprint publication and what they should look for.

Posting preprints is becoming increasingly popular. The DOI registrar Crossref reported that preprints were the fastest growing research output, at approximately $30 \%$, compared to an increase in articles by $2-3 \%$, in 2016-2018. ${ }^{1}$ There is even a preprint indexing service called PrePubMed. Therefore, it does not seem possible for scientific journals to stay out of this trend. In a study to evaluate the preprint publication policies of the 100 clinical journals with the highest impact factors, it was reported that $86 \%$ of them allowed submission of articles previously posted as preprints, and only one journal $(1 \%)$ prohibited the submission of preprinted manuscripts. $^{2}$ On the other hand, preprints still make up less than $3 \%$ of PubMed's monthly output. ${ }^{3}$
What is a preprint? COPE defines preprint as "a scholarly manuscript posted by the author(s) in an openly accessible platform, usually before or in parallel with the peer review process." 4 The purpose of preprints is to ensure that authors receive timely feedback and comments on research before it is submitted to a peerreviewed journal, to solicit the origin of an idea, and to facilitate and accelerate research dissemination and access to research.

What are the advantages and disadvantages of preprint? Speed, visibility, and priority are the major advantages. Moreover, as shown in a recent study, ${ }^{5}$ articles with preprints receive more citations than the ones without preprints. However, in some cases, these advantages of speed, visibility, and priority can turn into disadvantages. This is especially true in the health sciences, where presenting an article to the public without a peer-review process can potentially be harmful to public health. Table 1 has more details on this topic.

What do authors expect from the preprints? I think it would not be wrong to say that the authors' expectations of preprint are not being fully met. Fewer than $10 \%$ of preprints in biology and medicine receive comments, indicating that they do not tend to draw much attention. ${ }^{6}$ Another disappointing finding comes from the Krumholz et al. study, ${ }^{7}$ where they noted that only $10 \%$ of medRxiv preprints are ultimately published in a peer-reviewed journal. Table 2 has some more facts about preprints.

What do you need to know before submitting a preprint to a journal? There are some important points that authors should pay attention to. Does the journal have a preprint policy? What restrictions, if any, are there? What is required in preprint submission? What do you need to do as an author after acceptance? Table 3 has more information.

Publishing and rapid sharing of high-quality scientific information is our main goal. Establishing and meticulously implementing our preprint policy is an important step towards this goal. We encourage authors and scientists to submit their preprints to the Balkan Medical Journal. 
TABLE 1. Advantages and Disadvantages of Preprints

\section{Advantages \\ Speed: Preprints enable you to instantly share your research, without waiting weeks or months for peer-reviewed feedback. \\ Evolution: Preprints allow you to revise your manuscript before submitting it to a peer-reviewed journal. \\ Visibility: Preprints increase the visibility of your paper. \\ Priority: In a preprint, you can establish priority for your work, which is important for protecting your intellectual property rights and promoting your study. \\ Impact: In a fast-moving field, sharing your work in a preprint format can be a big advantage, as your research can have a greater impact on the direction of future studies. \\ Citation: Articles with preprints have a higher citation potential than the articles without preprints.}

\section{Disadvantages}

Speed and Peer Review: Publishing findings in a preprint format without a proper peer-review process has a risk of disseminating incorrect results, which may cause actual harm, especially in the medical sciences.

Novelty and Visibility: Some journals do not evaluate preprint publications as original work, and this may limit your journal choices, which will affect the visibility and impact of your article.

Priority: It has been argued that preprints expose your ideas, allowing the possibility of other researchers to "scoop" your work by writing and submitting it to a journal before you do.

TABLE 2. Some Facts About Preprints

- Preprints are growing at a rate ten times higher than journal articles. ${ }^{1}$

- Although the number of preprints appearing on bioRxiv has grown exponentially, they still make up less than $3 \%$ of PubMed's monthly output. ${ }^{3}$

- Articles with a preprint have $36 \%$ more citations than articles without a preprint. $^{5}$

- Less than $10 \%$ of the preprints in biology and medicine receive comments. ${ }^{6}$

- Only $9 \%$ of medRxiv preprints receive comments, and only $10 \%$ are published in peer-reviewed journals. ${ }^{?}$
TABLE 3. Some Important Things to Know Before Submitting Your Preprint to the Journal

- Does the journal have a preprint policy?

- Does the journal have any restrictions for preprint servers? (only nonprofit preprint servers allowed).

- Check the journal's preprint policy if the submitted manuscript needs to add meaningful new information in addition to the material in the preprint.

- Is there a DOI requirement to submit a preprint to the journal?

- Does the journal allow preprints to be used as references?

- Check if the preprint version is requested to be removed after the article is accepted by the journal?

- After publication, it is the authors, and not the journal, who are responsible for updating the archived preprint with the DOI and linking it to the published version of the article.

\section{Zafer Koçak®e}

Editor-in-Chief, Balkan Medical Journal Department of Radiation Oncology, Trakya University School of Medicine, Edirne, Turkey

\section{REFERENCES}

1. Lin J. Preprints growth rate ten times higher than journal articles. Crossref. 2018 (Available at: https://www.crossref.org/blog/preprints-growth-rate-ten-times-higher-t han-journal-articles/), Accessed June 18, 2021.

2. Massey DS, Opare MA, Wallach JD, Ross JS, Krumholz HM. Assessment of preprint policies of top-ranked clinical journals. JAMA Netw Open. 2020;3(7):e2011127. [CrossRef]

3. Penfold NC, Polka JK. Technical and social issues influencing the adoption of preprints in the life sciences. PLoS Genet. 2020;16(4):e1008565. [CrossRef]

4. COPE Council. Cope discussion document: preprints. publicationethics.org. 2018 (https://publicationethics.org/files/u7140/COPE_Preprints_Mar18.pdf), Accessed June 18, 2021.

5. Fu DY, Hughey JJ. Releasing a preprint is associated with more attention and citations for the peer-reviewed article. eLife. 2019;8:e52646. [CrossRef]

6. Leopold SS, Haddad FS, Sandell LJ, Swiontkowski M. Clinical orthopaedics and related research, the bone \& joint journal, the Journal of Orthopaedic Research, and The Journal of Bone and Joint Surgery will not accept clinical research manuscripts previously posted to preprint servers. J Bone Joint Surg Am. 2019;101(1):1-4. [CrossRef]

7. Krumholz HM, Bloom T, Sever R, et al. Submissions and downloads of preprints in the first year of medRxiv. JAMA. 2020;324(18):1903-1905. [CrossRef] 